\title{
Mobilité sédimentaire : définition par simulation des populations granulométriques mobiles sur l'avant-côte
}

\author{
Émile Akouango, Raphaël Certain et J. Paul Barusseau \\ LSM-CEFREM - Université de Perpignan - 52, av. de Villeneuve - 66860 \\ Perpignan
}

Résumé- Le but de ce travail est de rendre compte de la modification texturale accompagnant les évolutions granulométriques réelles observées sur les sables d'un environnement littoral.

La seule solution pratique consiste à simuler des enlèvements ou des arrivées de particules, appelés populations mobiles, selon une loi de fréquence déterminée.

A partir de la distribution expérimentale formalisée (première étape) et de la distribution de la population mobile ainsi définie (seconde étape), on détermine la distribution conséquente de façon à aboutir à une répartition qui coïncide, aussi précisément que possible, avec la distribution expérimentale observée (sédiment aval-transit ou sédiment post-coup de mer).

Plusieurs expériences ont été réalisées à partir d'échantillons provenant de situations réelles observées dans le cas de plages sableuses macrotidales, celles de Merlimont dans le Pas de Calais et d'Omaha Beach en Normandie. Les résultats sont présentés et discutés.

Abstract- This study aims at reporting textural changes linked with real grain size trend evolutions seen on sands in coastal environnement.

The only practical way consist in simulating removals or arrivals of particules, called movable populations, according to a law of determined frequency.

From the formalized experimental distribution (first stêp) and the distribution of a movable population thus defined (second step), the consequent distribution is determined to reach a repartition coinciding, as precisely as possible, with the experimental distribution observed (down drift sediment or post-storm sediment).

Several experiences have been realized from samples recovered in actual situations in the case of macrotidal sandy beaches, such as Merlimont in the Pasde-Calais or Omaha Beach in the Normandy. The results are presented and discussed.

\section{Granulométrie/ population mobile/modélisation/simulation}

\section{Introduction}

Il est largement admis que l'aptitude des sédiments à être remobilisés dépend de la distribution granulométrique complète des grains (McLaren et Bowles, 1985). L'action des écoulements lors des processus d'érosion modifie la distribution du sédiment initial par enlèvement d'une partie plus ou moins 
importante des grains qui le constituent. Les conditions du transport engendrent un spectre granulométrique du matériel déplacé et déterminent la distribution du flux des particules qui se déposent, modifiant par conséquent la texture du sédiment dans lequel se mélange cet apport. Dans la pratique, cependant, on continue d'utiliser un petit nombre de données, parfois même une seule (la médiane), pour caractériser ces distributions, ce qui a pour effet de conduire à des désaccords entre les observations et les prédictions de mouvement débutant, de transport et de dépôt. Ces désaccords sont d'autant plus grands que la distribution englobe un large spectre de particules. Des tentatives ont été faites pour entrer dans les modèles des représentations multi-classes du sédiment mais la durée des traitements informatiques oblige à en limiter l'application.

De nombreux auteurs ont cherché à analyser les changements progressifs qui marquent les différents états des sédiments au cours des phases de remaniement ou de dépôt (Haner, 1984). Cette reconnaissance est l'objet de la démarche de McLaren et Bowles (1985) pour tenter de modéliser les transformations auxquelles est soumis un sédiment défini par un ensemble de classes granulométriques. Ils ont supposé que les petits grains ont une probabilité de transport plus élevée que les grains de taille plus grande. Le phénomène peut être schématisé comme une suite d'extractions et d'apports quii tendent à transposer la forme de la distribution initiale vers celle de la distribution terminale par l'intermédiaire d'un flux exporté et/ou importé.

Gao et Collins (1991, 1994) ont exploité cette hypothèse en utilisant la variation des paramètres qui expriment les changements des caractéristiques granulaires au cours des processus d'érosion, de transport, de dépôt sélectif et de tri corrélatif. Ces paramètres de position (médiane) et de triage total (indice de dispersion) ou partiel (indice d'asymétrie) rendent compte de la «fraction sédimentaire mobile » (Ehrhold, 1999). Gao et Collins en tirent une méthode de détermination cartographique du transport sédimentaire par vecteurs. Elle ne donne toutefois aucune indication sur la population mobile dont le départ ou l'arrivée entraîne les changements paramétriques décrits. La détermination de cette population reste donc posée et, d'abord, celle de sa structure.

Il existe une grande incertitude sur la forme réelle de la distribution de ce matériel, lien entre deux états successifs du sédiment. La sélection des grains par les mécanismes de transport conduit à la construction de distributions sédimentaires considérées par les uns comme gaussiennes (Folk et Ward, 1957) ou comme des mélanges de populations normales (Moss, 1962). D'autres, avec McLaren et Bowles (1985), rappellent que le milieu empêche une parfaite adéquation de la distribution normale avec les distributions réelles en raison de la complexité des processus d'entraînement et de dépôt et de l'influence du matériel originel. Ce point de vue est justifié aussi par le fait que l'analyse et la compréhension des différences existantes sont rendues difficiles en raison du manque de connaissances qui s'observe, notamment dans le domaine des relations hydrosédimentaires (De Vriend, 1993).

Le but du présent travail est de rendre compte de la modification texturale accompagnant les évolutions granulométriques réelles observées dans un environnement marin, qui dans notre cas est caractérisé par des houles moyennes à faibles et la présence de marée. Pour cela, il convient: (1) de trouver une 
expression des courbes granulométriques réelles permettant l'interpolation de la valeur de fréquence associée à toute dimension de l'intervalle granulométrique considéré, (2) d'effectuer sur les distributions initiales ainsi formalisées des extractions ou des adjonctions de particules réparties, en première étape, selon des lois simples, (3) de vérifier, en utilisant une démarche d'essais et d'erreurs, que les paramètres des populations mobiles testées conduisent bien à l'obtention des distributions terminales réellement observées sur le terrain.

\section{Méthode}

\subsection{Principe}

Dans ce qui suit, on a supposé qu'à toute particule est associée une probabilité de mobilisation ou, inversement, de mise en place, dès lors que le critère de seuil minimum est dépassé. Cette probabilité s'exprime à travers la distribution des tailles de grains extraits ou apportés, formant une population mobile.

La différence entre les répartitions de fréquence des tailles de grains avant et après le phénomène responsable de la modification granulométrique résulte de l'exportation (érosion) ou de l'importation (apport, sédimentation) de cette population mobile: la comparaison directe des distributions granulômétriques du matériel transformé n'ouvre toutefois pas la possibilité de mettre en évidence la distribution de la population mobile. Cette voie conduit, en effet, à une impasse dans la mesure où, les représentations granulométriques étant relatives, les différences observées ne sont, en fait, que des artéfacts. Cette difficulté, qui n'a pas toujours été évitée (Thibault et Barusseau, 1975, Shih et Komar, 1990), est bien soulignée par McLaren et Bowles (1985).

La seule solution pratique consiste par conséquent à simuler, à partir d'une distribution originale connue, des enlèvements ou des arrivées de particules selon une loi de fréquence déterminée et de réduire le résultat au format relatif des courbes cumulatives en pourcentage. La courbe conséquente ainsi recalculée doit aboutir à une répartition qui coïncide, aussi précisément que possible, avec la distribution observée après la phase de transformation texturale. Pour cela, il convient de définir la loi de transformation granulométrique. McLaren et Bowles (1985) considèrent que la fonction de distribution des particules mobilisées augmente de façon monotone. Nous n'avons pas repris cette idée car des expériences variées (Barusseau, 1973) tendent à montrer que le matériel en transport présente une distribution à maximum, voisine d'une distribution de Gauss. La masse de particules extraites se réduit de part et d'autre d'un mode granulométrique caractéristique du phénomène d'érosion. On a donc admis en première étape, à la suite également des observations de Sengupta (1979), que lés populations mises en mouvement sont réparties selon une loi de Gauss. D’autres possibilités devront être testées ultérieurement (distribution de Rosin).

Dans chacun des tests réalisés, la distribution de la population mobile (en érosion ou en apport) a été définie par trois valeurs : (1) le mode $M$ de la population de grains extraite (population mobile), (2) son écart- type $\sigma$ et (3) 
l'ampleur P de la transformation, c'est-à-dire le pourcentage de matériel enlevé ou ajouté par rapport au matériel initial dans un intervalle de $\pm 2 \sigma$ autour de $M$.

Plusieurs séries de tests sont présentées en tenant compte de situations réelles observées dans le cas d'avant- côtes macrotidales soumises aux influences de la houle et de la marée.

\subsection{Provenance des échantillons}

Les échantillons étudiés proviennent de deux secteurs d'estran. Les prélèvements ont été réalisés sur les mêmes points de profils répétés dans le temps. Le matériel récolté représente le niveau superficiel $(0-1 \mathrm{~cm})$.

Le premier secteur se situe sur la plage intertidale de Merlimont (Pas de Calais, France), étudié dans le cadre d'une action spécifique de l'INSU, au printemps 1997.

Le second secteur est situé sur la plage intertidale d'Omaha Beach en Normandie à l'occasion d'un programme de recherche morphodynamique national (PNEC). Les échantillons ont été prélevés au printemps 1999.

Les caractères morphologiques et hydrodynamiques des deux secteurs sont identiques pour les périodes considérées. Ils présentent un relief commun à barres sédimentaires parallèles au rivage et l'agitation du plan'd'eau pendant l'étude dans les deux cas fut particulièrement faible (Levoy et al, 1998).

Les distributions granulométriques ont été obtenues par tamisage des échantillons lavés, sans plus ample traitement. Les teneurs en éléments bioclastiques sont généralement faibles $(<15 \%)$ et, dans la mesure où les matériaux récoltés sont fins dans les deux cas, on peut considérer que les écarts de forme des éléments coquilliers, par rapport aux grains quartzeux subsphériques, avaient peu d'importance dans le comportement hydrosédimentaire de l'ensemble des particules. Les valeurs corrigées des mailles de tamis ont été obtenues au microscope ou par tamisage d'un échantillon standard. Les dimensions sont indiquées en échelle des phi (cologarithme base 2 de la dimension en mm), seule façon de donner un sens à la notion d'écart-type pour des populations lognormales.

\section{Résultats}

\subsection{Changements granulométriques sur l'avant-côte de Merlimont}

Dans un premier temps, les caractéristiques générales des populations présentes sur la zone d'étude sont établies, puis l'exercice de simulation est exposé en prenant comme exemple celui effectué sur les échantillons de la barre intermédiaire entre le 25 et le 30 avril 1997 (comparaison temporelle) qui encadrent un événement de coup de mer modéré les 28 et 29 avril avec des hauteurs significatives maximales de 2,7 mètres, enfin les résultats globaux de l'ensemble des simulations sont présentés, donnant les caractéristiques des populations mobiles trouvées pour toute la période d'étude, en périodes de beau 
temps, de tempête (illustré par l'exemple décrit précédemment) et au cours d'un cycle de marée. Une synthèse graphique illustre l'exemple étudié.

(1) Les sédiments récoltés sur la plage intertidale de Merlimont sont des sables fins, bien à très bien classés dont le mode moyen pour le talus de haute plage est de $0,255 \mathrm{~mm}$ et celui des barres et bâches externes de $0,183 \mathrm{~mm}$ (Rapport final, 1997). On observe ainsi une décroissance des valeurs modales vers le large.

(2) L'exercice de simulation a été réalisé sur un intervalle de temps caractéristique en des points particuliers de la morphologie d'un estran présentant des barres sédimentaires. On présente ici un cas, celui de la simulation effectuée sur les échantillons de la barre intermédiaire entre le 25 et le 30 avril 1997, encadrant le coup de mer des 28 et 29 avril 1997.

La figure 1 représente les étapes successives de l'opération de modélisation réalisée sur les populations des 25 et 30 avril 1997 de la barre intermédiaire, à titre d'exemple illustratif de la méthode. Les deux premiers graphiques représentent différemment une même information, l'un montre les courbes cumulées en échelle semi-logarithmique et l'autre les courbes de fréquence. Ils illustrent la distribution réelle initiale (en trait plein) et la distribution réelle finale (en pointillé). L'objectif est, à partir de la distribution initiale, de retrouver la distribution finale, après un test d'ajout et/ou d'enlèvement de populations gaussiennes (respectivement GaussA et GaussE) considérées comme représentatives des fractions mobilisées entre les deux dates. Lorsque la courbe de distribution simulée correspond à la courbe finale réelle, la démarche a réussi. L'exercice démontre qu'il peut y avoir à la fois apport dans un intervalle granulométrique et simultanément érosion dans un autre intervalle.

(3) De la même manière, mais cette fois sur toute la période d'étude, l'ensemble des caractéristiques des populations mobiles obtenues par simulation est présenté dans les tableaux 1 et 2 . On peut en déduire les résultats suivants:

- II semble y avoir deux populations mobiles (aussi bien au cours de la tempête qu'au cours d'un seul cycle de marée ; aussi bien en érosion qu'en apport). L'unê est vers $220 \mu \mathrm{m}$, présentée pair le tableau 1. L'autre est vers $350 \mu \mathrm{m}$, présentée par le tableau 2.

- Les écarts-types sont généralement faibies. Les populations mobiles sont étroitement bornées autour d'une dimension centrale.

- L'hypothèse de distribution gaussienne des populations mobiles n'est pas juste, même si elle peut servir de première approche.

- La zone de la barre intermédiaire joue un rôle particulier dans les échanges lors des coups de mer. On a pu observer les populations mobiles mises en jeu sur cette dernière dans le cadre de l'exemple décrit précédemment (fig.1). On peut schématiser. l'ensemble de résultats obtenus au même moment sur l'ensemble de la plage intertidale (fig.2). La barre intermédiaire exporte donc une population mobile (fraction $0,22 \mathrm{~mm}$ ) qu'on retrouve dans les deux étages adjacents. Elle reçoit une population que délivre aussi bien l'étage supérieur que l'étage inférieur, ce qui est confirmé par Levoy (communication orale) qui a aussi observé à Omaha Beach (Calvados) un faible transport sédimentaire vers des zones immédiatement adjacentes. 
- Le matériel érodé en haut de plage (population mobile $0,35 \mathrm{~mm}$ ) se retrouve dans la zone des barres (fig.2).

- Il existe des échanges croisés entre sommet de barre et creux de bâche. En période de tempête, la barre perd des fins (fraction $0,22 \mathrm{~mm}$ ) et la bâche des grossiers (fraction $0,35 \mathrm{~mm}$ ). En période de restauration, l'inverse se produit, la barre perd des grossiers et la bâche des fins.

\subsection{Changements granulométriques sur l'avant-côte d'Omaha Beach}

Le sable d'Omaha Beach est un sable fin dont la médiane se situe autour de $0,2 \mathrm{~mm}$.

Comme dans le cas de Merlimont, l'évolution temporelle a été définie lors d'une période de beau temps, entre le 21 au 28 avril 1999. Cette approche étant peu concluante sur ce site, elle ne sera pas développée ici.

Une autre méthode consiste à comparer spatialement des échantillons adjacents sur un même profil. Des résultats ont ainsi été obtenus pour la journée du 28 avril 1999:

La comparaison spatiale du 28 avril 1999 met en évidence trois populations mobiles caractérisées respectivement par les valeurs centrales : 2,8 , 2,5 et $2 \varphi$, et observées depuis le haut de plage jusqu'à la limite externe de la plage intertidale. On remarque aussi que le matériel érodé en haut de plage (population 2,5 $\varphi$ ) est le même que celui apporté à l'avant-côte (fig.3). Les échantillons adjacents étant comparés du haut de plage vers le large, on remarque que la population mobile grossière $(2 \varphi)$ est de plus en plus importante du haut de plage vers la berme (fig.4) et de plus en plus faible de la berme vers le large. La population mobile fine $(2,5 \varphi)$ diminue, quant à elle, du haut de plage vers la berme, pour augmenter ensuite de la berme vers le large. La zone de la berme comprend donc la proportion la plus grande de population mobile grossière et le minimum de population fine. C'est l'illustration du caractère très dynamique de ce secteur.

Cette comparaison spatiale, ainsi que celle temporelle effectuée entre le 21 au 28 avril 1999, montre que les bâches perdent du matériel fin lors des périodes de beau temps, comme dans le cas de Merlimont.

\section{Discussion}

Malgré une certaine concordance des résultats, l'objet de cette démarche n'est pas d'essayer de mettre en valeur un cas général à partir des exemples étudiés mais de soulever des problématiques qui guideront cette recherche à parfaire. En particulier, l'approche spatiale utilisée dans le cas d'Omaha, et qui compare des échantillons adjacents sur un même profil à un moment identique du cycle de marée, devra être appliquée à Merlimont.

Le premier fait marquant est la mise en valeur des échanges entre la hauteplage et la zone des barres. La simulation granulométrique met en évidence une population mobile érodée de la haute-plage, identique à celle apportée vers l'avant-côte dans les deux cas étudiés. Il semblerait donc que cette méthode 
permette une bonne mise en évidence des échanges qualitatifs entre les différents compartiments.

Le second point est la perte de matériel fin dans les bâches lors des périodes de beau temps. Simultanément, la perte de matériel grossier par les barres ne se vérifie seulement que dans un cas. Ce résultat pourrait mettre en évidence une uniformisation de la granulométrie sur l'ensemble de l'avant-côte lors des périodes de beau temps peut-être par le phénomène de migration des barres vers le rivage.

Le résultat le plus frappant est l'appartenance des populations mobiles à deux ou trois groupes granulométriques fixes quelque soit leur positionnement sur la zone d'étude. Cette typologie restreinte du matériel hypothétique participant aux échanges et son indépendance vis-à-vis de la profondeur pourrait indiquer l'action du balayage de la zone intertidale par le point de déferlement lors du jusant. Les changements granulométriques enregistrés seraient alors la marque du passage d'un seul et même événement dynamique.

\section{Conclusion}

Les résultats obtenus lors de cette étude mettent en valeur certaines pistes de recherche qui méritent d'être approfondies. Parallèlement la méthode devra être améliorée par le biais notamment de tests en canal à houle pour apporter à cette modélisation le côté quantitatif qui lui manque.

\section{BIBLIOGRAPHIE}

Barusseau J.P. 1973. Evolution du plateau continental rochelais (Golfe de Gascogne) au cours du Pléistocène terminal et de l'Holocène. Les processus actuels de la sédimentation. Thèse non publiée, Université de Bordeaux, 363 p. + ann.

Barusseau, J.P., Diouf, M.B., de la Bardonnie, M., El Ghandour, N., 1998. Méthodologie pour une simulation des transformations granulométriques de sables de la zone d'avant-côte. Oceanologia Acta, 22, 2, 179-191.

De Vriend H. J. 1993. G6/G8 coastal morphodynamics : introduction. Mast Days and Euromar Market, 15-17 March 1993, Part II : Coastal Morphodynamics and Structures, Project Reports, vol. 2, 441-463.

Ehrhold, A., 1999. Dynamique de comblement d'un bassin sédimentaire soumis à un régime mégatidal : un exemple de la Baie du Mont-Saint-Michel, thèse de doctorat, Univ.de Caen, 293p. + ann.

Folk R.L. and Ward W.C. 1957. Brazos River bar : a study in the significance of grain-size parameters. Journal of Sedimentary Petrology, 27, 3-26.

Friedman G.M. 1967. Dynamic processes and statistical parameters compared for size frequency distribution of beach and river sands. Journal of Sedimentary Petrology, 37, 327-354.

Gao $\mathrm{S}$ and Collins M. 1991. A critique of the "McLaren method" for defining sediment transport paths. Joumal of Sedimentary Petrology, 61, 143-146. 
Gao S. and Collins M. 1994. Analysis of grain-size trendsfor defining sediment transport pathways in marine environments. Journal of Coastal Research, 10, 1, 70-78.

Haner B.E. 1984. Santa Ana River : an example of a sandy braided floodplain system showing sediment source area imprintation and selective sediment modification. Sedimentary Geology, 38, 247-261.

Levoy, F., Anthony, E., Barusseau, J.P., Howa, H. et Tessier, B., 1998. Morphodynamique d'une plage macrotidale à barres. C.R. Acad. Sci., Paris, Sciences de la terre et des planètes, 327, 811-818.

McLaren P. and Bowles D. 1985. The effects of sediment transport on grain-size distributions. Journal of Sedimentary Petrology, 55, 454-470.

Moss A.J. 1962. The physical nature of common sandy pebbly deposits, part I. American Journal of Sciences, 260, 337-373.

Rapport final, Action spécifique de recherche, 1997. Echanges sédimentaires plage- prisme subtidal, rapport non publié, $44 \mathrm{p}$.

Sengupta S. 1979. Grain-size distribution of suspended load in relation to bed materials and flow velocity. Sedimentology, 26, 63-82.

Shih S.M. and Komar P.D. 1990. Hydraulic controls of grain-size distributions of bedload gravels in Oak Creek, Oregon, USA. Sedimentology, 37, 377-384.

Thibault M. and Barusseau J.P. 1975. Deux exemples des modalités de triage granulométrique sur le littoral et l'avant-côte.Proceedings of the IXth International Congress of Sedimentology, Nice, 6, 205-210. 

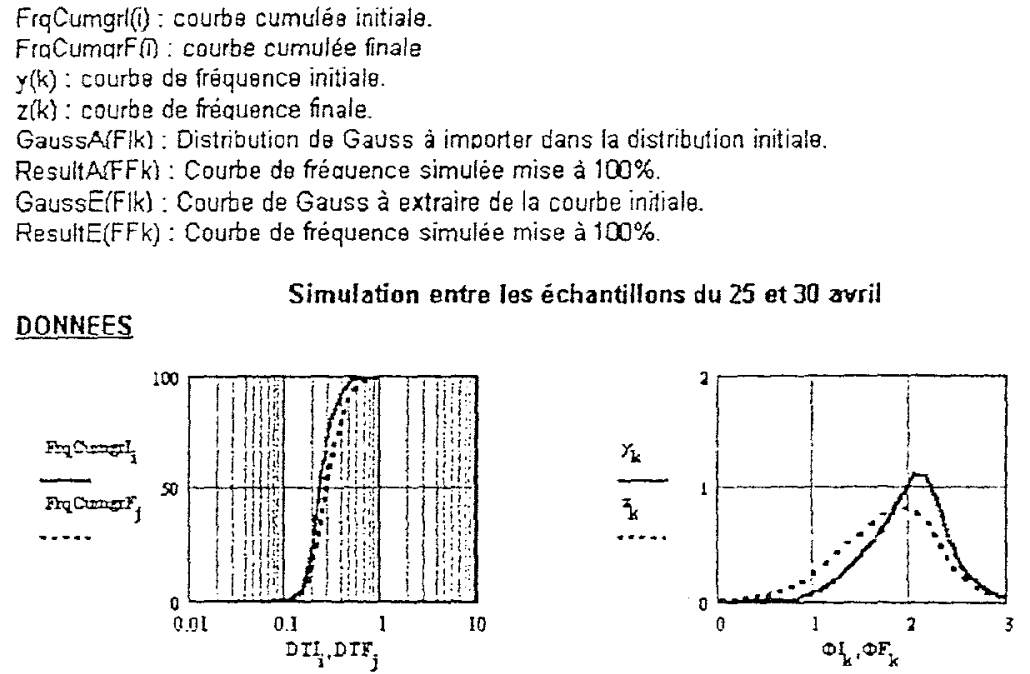

RESULTATS ARRES ARNORT TLÜLEA $=1 . . \mathrm{SS}$

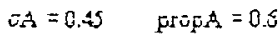
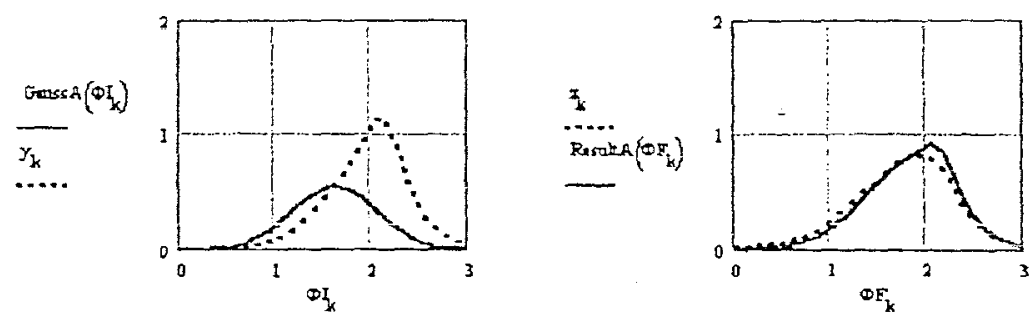

RESULTATS APRES EROSION

$$
\begin{array}{lll}
\text { roode } A=1.65 & o \mathrm{~A}=0.45 & \text { prop } A=0.6 \\
\text { roode } E=2.15 & \mathrm{oE}=0.2 & \text { propE }=0.1
\end{array}
$$
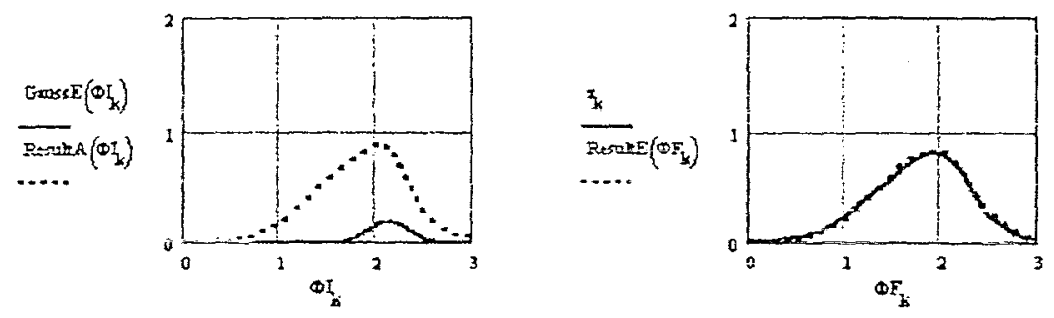

Fig. 1 : Simulation du passage entre une distribution réelle initiale (sédiment avant érosion et apport extérieur) et une distribution réelle finale (sédiment après érosion et apport extérieur) par introduction de populations gaussiennes.

Exemple de la simulation effectuée sur la barre intermédiaire entre le 25 et le 30 avril 1997 à Merlimont.

Simulation of the transition between an initial actual distribution (sediment before erosion and external deposit) to find the final actual distribution (sediment after erosion and after deposit) by adding and removing of nomal populations. Exemple of the simulation realized on the middle bar in Merlimont between the aprit 25 th and the april 30 th 1997. 


\begin{tabular}{|c|c|c|c|c|}
\hline Dim. Moy. & Dim. Min. & Dim. Max. & $\begin{array}{c}\text { Écart-type } \\
\text { (phi) }\end{array}$ & prop (\%) \\
\hline 225 & & & 0,2 & -10 \\
\hline 220 & 175 & 275 & 0,32 & +85 \\
\hline 220 & 195 & 240 & 0,15 & +25 \\
\hline 205 & 185 & 220 & 0,13 & +45 \\
\hline 225 & 195 & 260 & 0,2 & -25 \\
\hline 185 & 170 & 200 & 0,12 & +85 \\
\hline 195 & 170 & 225 & 0,2 & +55 \\
\hline 215 & 175 & 265 & 0,3 & +65 \\
\hline 235 & 195 & 275 & 0,25 & +20 \\
\hline 205 & 170 & 240 & 0,25 & +5 \\
\hline 220 & 185 & 260 & 0,25 & -20 \\
\hline 185 & 155 & 220 & 0,25 & +35 \\
\hline 175 & 160 & 195 & 0,14 & +70 \\
\hline 230 & 210 & 255 & 0,14 & +50 \\
\hline 180 & 165 & 195 & 0,13 & -45 \\
\hline 235 & 205 & 270 & 0,2 & -45 \\
\hline
\end{tabular}

Tabl.1 : Ensemble des résultats correspondant à la population mobile $220 \mu \mathrm{m}$ (la fraction mobile de l'exemple étudié est représentée en gras)

Set of results corresponding to the movable population 220 um (the movable population of the examined example is in bold)

\begin{tabular}{|c|c|c|c|c|}
\hline Dim. Moy. & Dim. Min. & Dim. Max. & $\begin{array}{c}\text { Écart-type } \\
\text { (phi) }\end{array}$ & prop (\%) \\
\hline 319 & & & 0,45 & +60 \\
\hline 320 & 275 & 370 & 0,20 & -70 \\
\hline 380 & 355 & 405 & 0,10 & -5 \\
\hline 320 & 270 & 380 & 0,25 & -43 \\
\hline 355 & 275 & 450 & 0,35 & +55 \\
\hline 340 & 270 & 435 & 0,35 & -50 \\
\hline 340 & 265 & 435 & 0,35 & -20 \\
\hline 365 & 270 & 500 & 0,45 & -20 \\
\hline 380 & 285 & 500 & 0,40 & -3 \\
\hline 355 & 300 & 420 & 0,25 & +10 \\
\hline 450 & 380 & 535 & 0,25 & +10 \\
\hline 275 & 225 & 340 & 0,30 & -50 \\
\hline
\end{tabular}

Tabl.2 : Ensemble des résultats correspondant à la population mobile $350 \mu \mathrm{m}$. (la fraction mobile de l'exemple étudié est représentée en gras) Set of results corresponding to the movable population 350 um (the movable population of the examined example is in bold) 


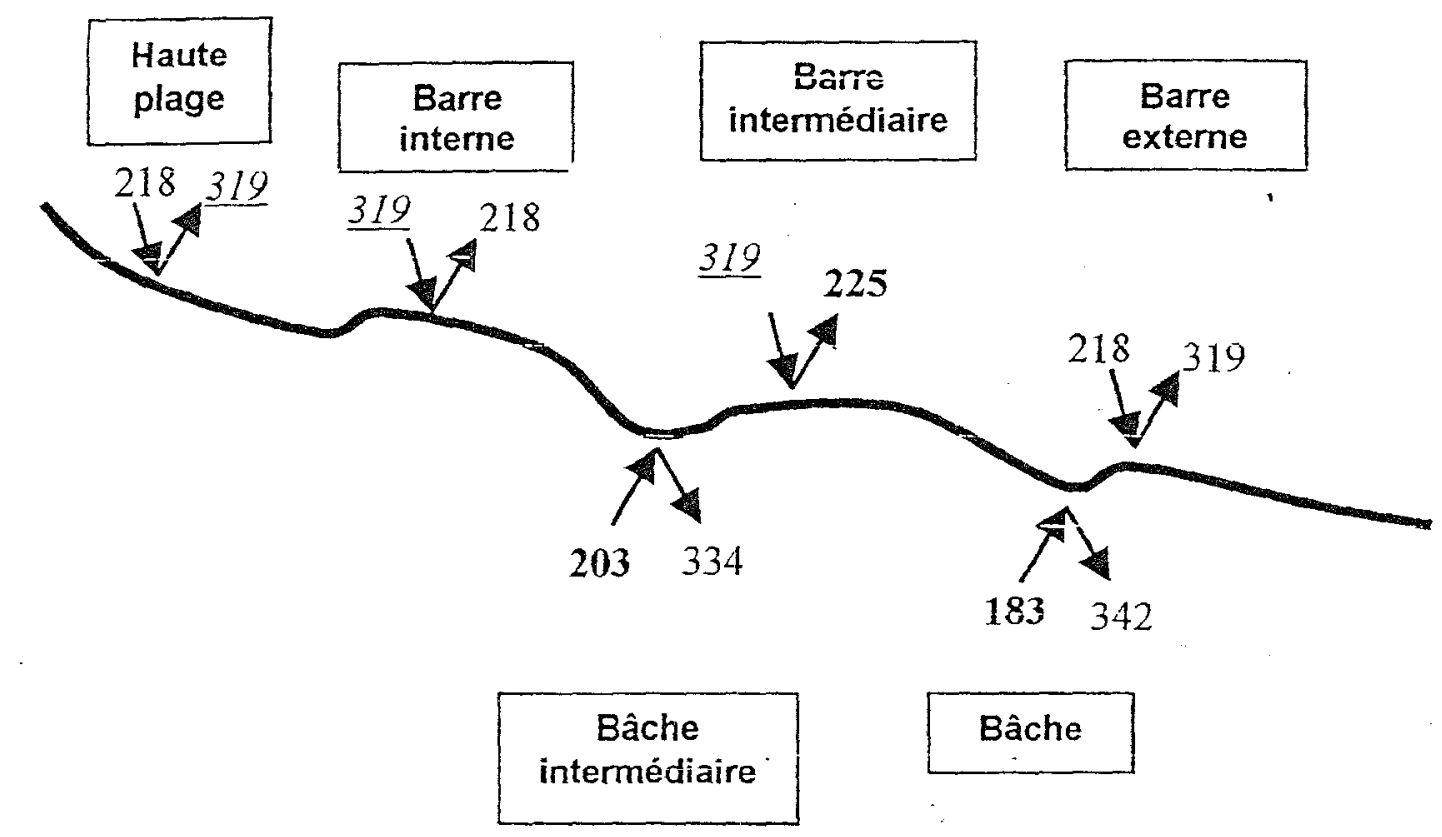

Fig.2 : Schéma des échanges des populations mobiles au cours de la tempête du 28-29 avril 1997 à Merlimont.

En gras, les échanges barre intermédiaire/bâches. Souligné, les échanges haut de plage/plage intertidale. Flèche vers le profil : apport. Flèche à l'opposé du profil : érosion.

Diagram of the exchange among the movable population during the $28-29$ april 97 storm in Merlimont. In bold, the exchanges middle between ridge and Irunnel. Underlined, the exchanges between the top of the beach and the intertidal beach. Arrow toward the profile: imput. Arrow opposite to the profile : oulpul. 


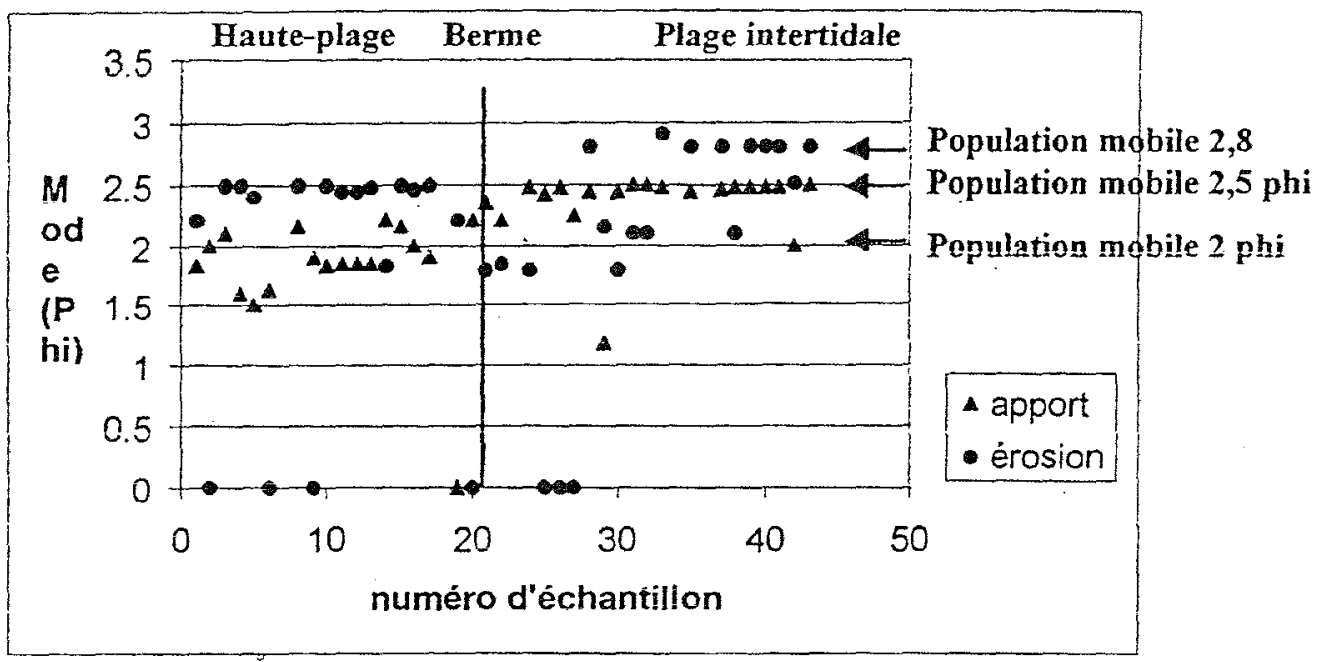

Fig.3 : Populations mobiles apportées et érodées le 28/05/99 à Omaha Beach. Les échantillons sont en ordre croissant du haut de plage vers la limite externe de la plage intertidale. L'échantïlon $n^{\circ} 20$ correspond à la berme.

Movable population imput and output at Omaha beach on 28/05/99. The sample numbers are increasing from the top of the beach to the external limit of the intertical beach. Sample rumber 20 corresponds to the berm.

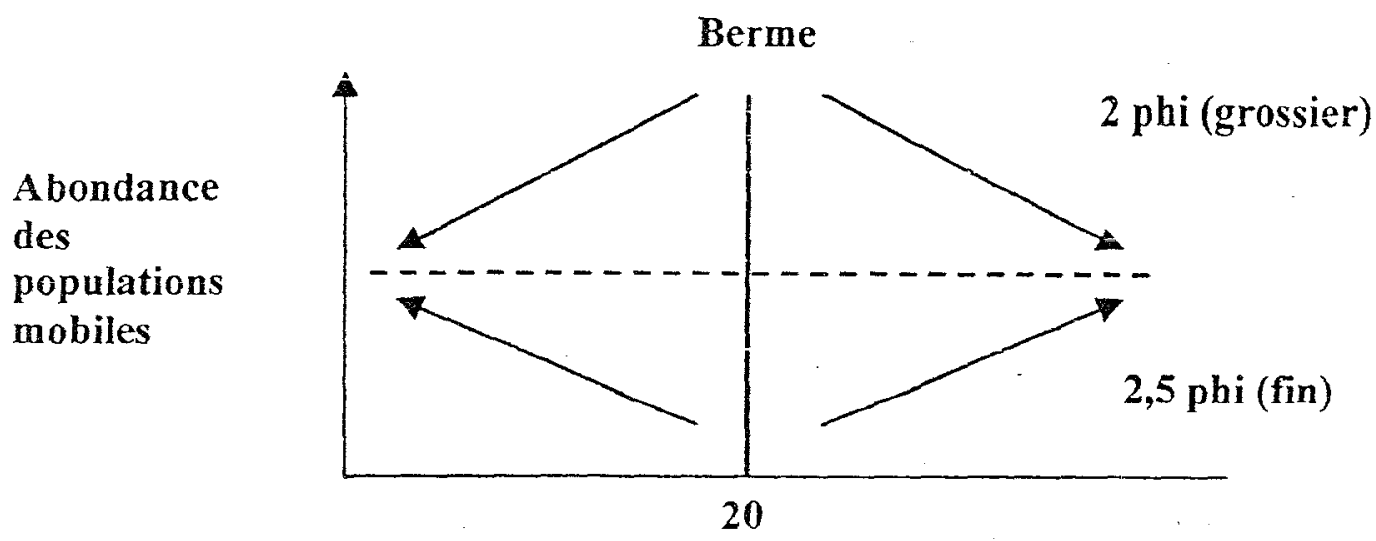

\section{Numéro d'échantillon}

Fig.4 : Abondances respectives des deux fractions mobiles fines et grossière en fonction de leur localisation sur le profill.

The respective abundance of the two movable populations (fine and coarse) according to their position on the profile. 